\title{
Estado actual de las investigaciones etnobotánicas en México
}

\author{
MIGUEL ANGEL MARTINEZ ALFARO \\ Jardín Botánico, Instituto de Biología, UNAM. Apdo. Postal 70-614, Coyoacán. 04510, México, D. F.
}

\begin{abstract}
Resumen. Este trabajo presenta la situación actual de la etnobotánica en México, con algunos estudios notables llevados a cabo en años recientes o que están en proceso. Presentamos alguna información general para la etnobotánica latinoamericana que sirva como punto de comparación en el desarrollo de esta disciplina para esta parte del mundo. Así mismo, para una mejor comprensión de la etnobotánica y su orientación multidisciplinaria, se proporcionan comentarios sobre aspectos epistemológicos y los enfoques cualitativos y cuantitativos.
\end{abstract}

Abstract. This paper shows the actual situation of the ethnobotany in Mexico, with some notable studies carried out in recent years or in process. We present some general information for the ethnobotany in Latin America as comparative point in the develoment of this discipline. Comments are given in epistemogical aspects and the qualitative or quantitative approaches for better explanation of the ethnobotany and its multidisciplinary orientation.

\section{INTRODUCCIÓN}

La etnobotánica es un campo de la ciencia con un carácter multidisciplinario, que estudia las relaciones entre las sociedades humanas y las plantas, y cuyo campo de acción es muy amplio en lo concerniente a los temas que trata. Estas características, además del hecho de que es una disciplina nueva, dificultan su definición y la delimitación de su objeto de estudio, alcances, utilidad, y sobre todo su ubicación como ciencia.

En virtud de no contar en un sentido ortodoxo y estricto con una teoría y método propios, paradigmas sólidos y una consolidación definitiva como especialidad botánica, todo parecería indicar que su verdadera utilidad teórica o práctica es casi nula.

Tal inicio al presente artículo es justamente para mostrar los logros en este campo de la ciencia que sigue avanzando, contra lo que piensan algunos biólogos y filósofos de la ciencia, y cada vez son más los centros de investigación biológica que manejan programas de esta disciplina. Además su popularidad es creciente en organismos internacionales tales como la Unión Internacional de Conservación de la Naturaleza (UICN), la Organización para la Agricultura y Alimentación (FAO-NU); la Organización Mundial de la Salud (WHO-NU), la Fundación Mundial para la Vida Silvestre (WWF), por citar algunas de las más importantes, las cuales solicitan cada vez mayor apoyo de la etnobotánica.

Es obvio que tal popularidad de la etnobotánica se debe a su utilidad y a que se sustenta en un marco teórico sólido que tiene un enfoque multidisciplinario, lo que algunos epistemólogos y filósofos de la ciencia consideran como una materia común en una época de síntesis de las disciplinas, es decir en un momento de ciencia no formal según lo define Kuhn (1979).

\section{LA ETNOBOTÁNICA DENTRO DE LAS CIENCIAS BIOLÓGICAS CONTEMPORÁNEAS}

En la actualidad la ciencia se desarrolla con una tendencia a formar nuevos campos del conocimiento. Los desarrollos en algunas disciplinas invitan a buscar la síntesis de varias especialidades, ya sea al interior de una ciencia o de varias. Esto ocurre dentro de la etnobotánica, en donde campos de estudio tales como la etnoecología, la ecología humana, la conservación de recursos naturales, la economía de recursos naturales, etc., se ven obligados a compartir teorías y métodos de investigación para enfrentar problemas complejos. La vinculación de disciplinas diferentes obedece a que hay investigaciones y/o problemas de investigación que obligan a salirse del modelo o cuadro de las especialidades individuales y crear nuevas disciplinas. Según el tipo de síntesis de disciplinas que se haga, se van formando nuevas líneas de investigación etnobotánica.

Este proceso por el que pasa la ciencia moderna lleva a la formación de nuevos enfoques teóricos y mejoras metodológicas, que a la vez cambian los paradigmas en los que se encuentra un campo científico. Siguiendo a Kuhn (1979) y a Harré (1970), los paradigmas se pueden ver desde tres puntos de vista o niveles: el del grupo de especialistas o profesionales de la disciplina o campo científico, el de los modelos que se aplican al estudio de las líneas de investigación que enfrenta la especialidad, y finalmente, el del desarrollo teórico máximo al que llega la ciencia. En el caso de la etnobotánica los dos primeros niveles se encuentran ya desarrollados y el tercero está construyéndose.

Otro aspecto que ayuda a situar con más claridad el quehacer del etnobotánico es el referente a cómo concibe la ciencia un grupo de científicos, en nuestro caso los botánicos. Estamos en un país donde los parámetros para medir la calidad e impacto de la ciencia se definen a través del modelo 
positivista, el cual privilegia la cuantificación sobre la descripción. Este aspecto se entiende mejor cuando se consideran los niveles sociales de la ciencia. Algunos autores como Price (1965, en Holt y Schoorl, 1993) llaman a estos niveles sociales «estados de la ciencia». Desde su punto de vista se pueden distinguir cuatro niveles: el político, el administrativo, el profesional-educativo y el técnico-científico. Ninguna ciencia escapa de ser evaluada desde los cuatro niveles o estados. En cada uno de ellos se le juzga de manera diferente y se le otorga una presencia o peso distinto. Así existen ciencias en las que vale más el aporte aplicado que el teórico. En otras su valor político puede ser lo principal, o sus cuerpos profesionales, etc., Esto también se aplica a las ciencias o campos de la ciencia en formación.

A la par del esquema propuesto por Price, existe una base teórica para distinguir los diferentes alcances y papeles que juega cada estado de la ciencia en una sociedad. Aquíse adopta la interpretación ofrecida por Burrell y Morgan (1979 en Holt y Schoorl, 1993) concerniente a cómo la sociedad mira a la ciencia, en particular cómo un grupo de científicos observa a sus colegas (Fig. 1). Así tenemos que hay dos ejes en los que se mueven los científicos, uno que va de lo subjetivo a lo objetivo y otro que va de la crítica a la aceptación de una ciencia en el contexto social y profesional. Una ciencia será más madura mientras más caiga en el cuadrante de lo objetivo y aceptado, y mientras tenga un impacto mayor entre los científicos, o entre amplios sectores del conjunto social. Cabe mencionar que la cuantificación no debe ser necesariamente el principal criterio para considerar más objetiva y aceptada una disciplina. Estas notas introductorias tienen el objetivo de evitar ideas equivocadas o prejuicios hacia un campo de la ciencia complejo y que está sujeto a la crítica de distintas corrientes filosóficas que existen en las ciencias.

Lo anterior no indica que la etnobotánica deba ser principalmente descriptiva. Muchos de los modelos matemáticos derivados de los que usan los ecológos en el análisis de poblaciones, comunidades y ecosistemas son sumamente útiles en este tipo de estudios. Además, la etnobotánica logra una importante proyección cuando se relaciona con la ecología vegetal; las dos especialidades han alcanzado importantes avances cuando se relacionan con investigaciones cuyo centro de análisis es la relación sociedad y ecosistemas, logrando enfoques más ricos que los obtenidos por la etnobiología, la etnoecología o la ecología humana por sí solas.

La madurez de la etnobotánica se alcanzará en el momento en que las descripciones o los análisis cuantitativos ayuden a establecer los modelos teóricos más adecuados en el estudio de las relaciones: sociedades humanas-naturaleza.

Por otro lado, al ser la etnobotánica un campo multidisciplinario está limitada por la disciplina desde la que se hace la investigación. Sólo menciono tal situación, dado que en estas notas me restringiré al campo botánico. Los estudios sobre conservación de recursos fitogenéticos y su mejoramiento constituyen una de las líneas multidisciplinarias de mayor importancia. También lo son los trabajos de

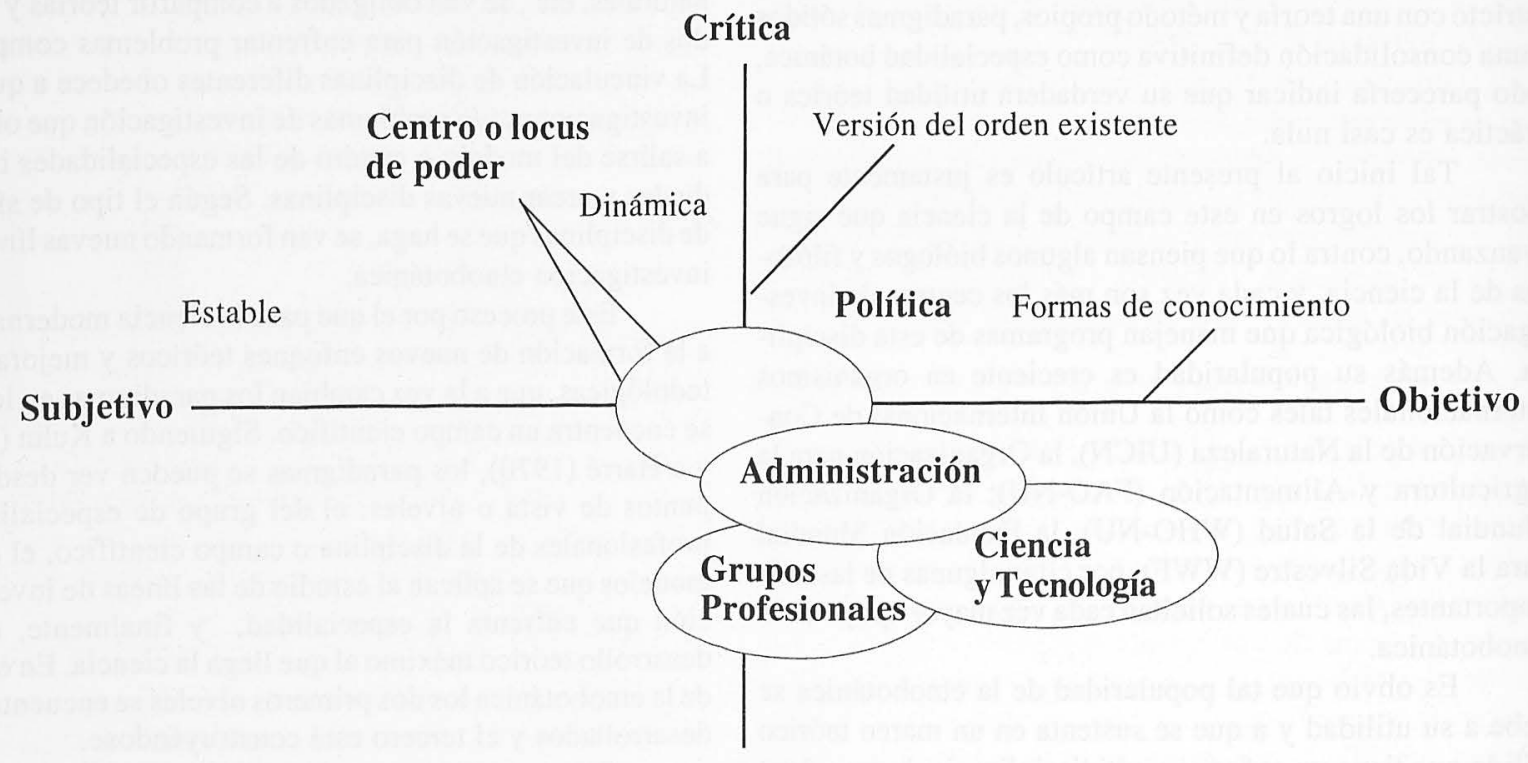

Aceptación

FIgura. 1. Una tipología «ideal» de las características y papeles o funciones de los cuatro estados de la ciencia (según Holt y Schoorl, 1993) 
evolución bajo domesticación, clasificación y sistemática de plantas cultivadas, orígenes de la agricultura y evolución de sistemas agrícolas; los estudios sobre percepción, clasificación y manejo de los recursos vegetales por un grupo humano; los estudios cuali y cuantitativos sobre floras médicas y comestibles; las investigaciones sobre cultivo y difusión de razas criollas o nativas de interés para la economía local y los trabajos sobre conservación in y ex situ de plantas cultivadas o de sus parientes más próximos.

\section{SITUACIÓN ACTUAL DE LA ETNOBOTÁNICA EN LATINOAMÉRICA Y MÉXICO}

Una de las características actuales de la etnobotánica es la búsqueda de campos de la enseñanza de la botánica en los que el alumno aprenda a manejar problemas de investigación con enfoques novedosos, e integre los conocimientos generados en las diversas áreas de la biología contemporánea. Por ello deben tener especial consideración los rápidos cambios tecnológicos que vivimos en la cibernética, en la misma biología, en genética molecular, con el desarrollo de técnicas y equipo de laboratorio y de trabajo de campo; la ecología en sus diversos ámbitos; la epistemología y otros campos de la filosofía de las ciencias. Todo esto ha permitido la definición de nuevos campos de las ciencias naturales, ya que ha habido y hay desarrollo en la teoría y por consiguiente en los métodos. Ante esta situación se presentan nuevos problemas a investigar y existen también nuevos enfoques en el análisis de los problemas conocidos. Como bien indica Kuhn (1979) existe una revolución científica en proceso que puede pasar inadvertida en nuestros círculos científicos, en las universidades y centros de investigación si no estamos atentos al desarrollo epistemológico y filosófico de la biología y menos aun en otras disciplinas. Pareciera natural aplicar tal o cual técnica o método sin analizar el contexto social que lo origina y/o aplica. Así lo advierten el físico Harré (1970) y el matemático Koyre (1983), que son además filósofos de la ciencia y estudiosos del desarrollo científico actual.

Otra situación que favorece materias como la que aquí nos ocupa es que aunque no se han creado grupos de investigación en los países en vías de desarrollo, en la actualidad se invierten cada vez más fondos para la ciencia y se van formando recursos humanos que respondan a las demandas del mundo moderno.

En los países del primer mundo la etnobotánica es vista con dos perfiles: a) es una materia que estudia los usos pasados, curiosos y raros de las plantas, y su utilidad radica en que esa antigua sabiduría es o puede ser incorporada a las necesidades biológicas o culturales del mundo actual; y b) la etnobotánica es una disciplina que advierte sobre el peligro del deterioro ambiental, y que permite apreciar cómo sociedades no complejas saben administrar mejor el manejo del ambiente, además de que tales grupos habitan regiones con alta biodiversidad, lo que puede sugerir un modelo de manejo ambiental al que muchos llaman sustentable; en esas regiones se pueden encontrar soluciones a demandas de tal o cual vegetal y son reservorios de recursos para la humanidad. Así es como el estudio etnobotánico es la llave para entrar a un mundo donde la diversidad biológica y cultural debe ser preservada. En otras palabras, hay un discurso político, económico y científico que demanda el primer mundo para justificar sus presiones e intromisiones y para dictar políticas científicas acerca de lo que es bueno para los pobres. Por lo general, el etnobotánico estadounidense, británico o francés se interesa mucho en el rescate de las plantas y la sabiduría popular, pues ante el cambio cultural hay un inminente proceso de eliminación y desaparición de conocimientos populares que pueden ser útiles para el hombre civilizado.

En los países que están en vías de desarrollo o que son dependientes de las naciones hegemónicas, la etnobotánica también es vista con dos orientaciones: a) como disciplina redentora, que busca ir al rescate de lo que se va a extinguir; b) como un campo de aplicación para el buen manejo o la conservación de los recursos vegetales, dándole un lugar privilegiado al conocimiento popular que lo integre al que tiene el botánico, y buscando soluciones entre el investigador y el grupo investigado. Aquí se ve otra actitud ética ante la investigación. Esta situación lleva, por un lado, a que el trabajo etnobotánico se presente como muy subjetivo, socializado y discursivo. Por otro lado, se le mira como algo anecdótico y sentimentaloide, cosas que desafortunadamente suelen ocurrir y que le dan a la etnobotánica un elemento más para ser criticada. Si al cuadro anterior le añadimos que mucha gente interesada en etnobotánica no sabe botánica sistemática, más desprotegidos quedamos ante la mirada analítica e inquisidora de los colegas de otros campos de la botánica. La etnobotánica se configura ya como una ciencia holística y según quien la estudie tendrá mayor o menor rigor científico, de acuerdo al desarrollo epistemológico o teórico de las disciplinas que la conforman.

En el caso de México se formó una corriente sólida que busca ser copiada o seguida por botánicos de Latinoamérica u otras partes del mundo. Se tienen diversos y buenos estudios, se puede ofrecer más calidad y diversidad de trabajos, enfoques y métodos a seguir. La teoría, en mi opinión, no es limitada. Es en los trabajos y los métodos donde se debe contar con mejores herramientas, entre ellas están las que ofrecen los métodos experimental, el estadístico y el comparativo.

Las disciplinas en que se apoya mucho la etnobotánica son: taxonomía, ecología vegetal, genética, la evolución vegetal, fitogeografía, físiología de plantas y la florística. Además de varias especialidades de la agronomía, la antropología, la química y la historia; todas confluyen en este campo de la ciencia, de ahí su importancia actual pues hay diversos enfoques y disciplinas desde los cuales puede abordarse su estudio. 
Cada vez hay más cursos e investigaciones etnobotánicas en diversas instituciones educativas y de investigación en Latinoamérica y por lo tanto en México. No se ven limitantes para el desarrollo de esta disciplina, los límites los ponen los propios investigadores y por un buen tiempo la etnobotánica orientada al estudio de las plantas medicinales seguirá dominando el panorama de la etnobotánica a nivel mundial y de nuestro país. A continuación se presenta un cuadro donde se muestran las líneas de investigación etnobotánica que se realizan en América Latina y se comparan con México (Martínez, 1991). Se presentan las estadísticas por país y el tipo de investigador, si es nacional $(\mathrm{N})$ o extranjero $(\mathrm{E})$ (Cuadro 1).

El Cuadro 1 presenta el estado actual de las investigaciones etnobotánicas en México y Latinoamérica con el objeto de que sirva como elemento de comparación. Así

CUADRo 1. Líneas de investigación etnobotánica que se realizan en América Latina.

\begin{tabular}{|c|c|c|c|c|c|c|c|c|c|c|c|}
\hline PAIS & $\begin{array}{l}\text { Arqueo- } \\
\text { botánica }\end{array}$ & $\begin{array}{l}\text { Plantas } \\
\text { medi- } \\
\text { cinales }\end{array}$ & $\begin{array}{l}\text { Plantas } \\
\text { comes- } \\
\text { tibles }\end{array}$ & $\begin{array}{l}\text { Estudios } \\
\text { cogno- } \\
\text { sitivos }\end{array}$ & $\begin{array}{l}\text { Aprovecha- } \\
\text { mientos } \\
\text { forestales }\end{array}$ & $\begin{array}{l}\text { Sistemas } \\
\text { agrosil- } \\
\text { vicolas } \\
\text { y huertos }\end{array}$ & $\begin{array}{l}\text { Domesti- } \\
\text { cación y } \\
\text { origen de la } \\
\text { agricultura }\end{array}$ & $\begin{array}{l}\text { Estudios } \\
\text { históricos }\end{array}$ & Mercados & $\begin{array}{l}\text { Gene- } \\
\text { rales }\end{array}$ & Totales \\
\hline $\begin{array}{l}\text { Antillas } \\
\text { Holandesas }\end{array}$ & & $11(\mathrm{E})$ & $4(E)$ & $1(E)$ & $1(E)$ & & & $2(E)$ & & $\begin{array}{l}1(\mathrm{~N}) \\
3(\mathrm{E})\end{array}$ & 23 \\
\hline Argentina & $\begin{array}{l}2(\mathrm{~N}) \\
2(\mathrm{E})\end{array}$ & $\begin{array}{r}10(\mathrm{~N}) \\
3(\mathrm{E})\end{array}$ & $15(\mathrm{~N})$ & $\begin{array}{l}4(\mathrm{~N}) \\
4(\mathrm{E})\end{array}$ & $\begin{array}{l}1(\mathrm{~N}) \\
10(\mathrm{E})\end{array}$ & $3(\mathrm{~N})$ & $\begin{array}{r}21(\mathrm{~N}) \\
8(\mathrm{E})\end{array}$ & & & $7(N)$ & 90 \\
\hline Bahamas & & $7(\mathrm{E})$ & & & & & $1(\mathrm{E})$ & & & $2(\mathrm{E})$ & 10 \\
\hline Barbados & & $2(E)$ & 2(E) & & & 2(E) & 2(E) & & & $3(E)$ & 11 \\
\hline Belice & $36(E)$ & $3(E)$ & $1(E)$ & $1(E)$ & $1(E)$ & $1(E)$ & $3(E)$ & $2(E)$ & & $3(E)$ & 51 \\
\hline Bermudas & & $1(\mathrm{E})$ & & & & & & & & & 1 \\
\hline Bolivia & & $\begin{array}{c}12(\mathrm{E}) \\
1(\mathrm{~N})\end{array}$ & $\begin{array}{l}2(\mathrm{~N}) \\
5(\mathrm{E})\end{array}$ & $1(\mathrm{~N})$ & 1 & $2(\mathrm{E})$ & $\begin{array}{r}10(\mathrm{~N}) \\
8(\mathrm{E})\end{array}$ & & & $1(E)$ & 43 \\
\hline Brasil & $\begin{array}{l}4(\mathrm{~N}) \\
1(\mathrm{E}) \\
\end{array}$ & $\begin{array}{r}10(\mathrm{~N}) \\
9(\mathrm{E}) \\
\end{array}$ & $\begin{array}{l}7(\mathrm{~N}) \\
6(\mathrm{E}) \\
\end{array}$ & $\begin{array}{l}1(\mathrm{~N}) \\
1(\mathrm{E})\end{array}$ & $\begin{array}{l}7(\mathrm{~N})) \\
3(\mathrm{E})\end{array}$ & $\begin{array}{l}4(\mathrm{~N}) \\
2(\mathrm{E})\end{array}$ & $\begin{array}{r}20(\mathrm{~N}) \\
4(\mathrm{E})\end{array}$ & & $1(\mathrm{~N})$ & $\begin{array}{l}6(N) \\
3(\mathrm{E})\end{array}$ & 90 \\
\hline Chile & $\begin{array}{l}6(\mathrm{~N}) \\
1(\mathrm{E})\end{array}$ & $\begin{array}{r}12(\mathrm{~N}) \\
2(\mathrm{E})\end{array}$ & $\begin{array}{l}17(\mathrm{~N}) \\
10(\mathrm{E})\end{array}$ & & $\begin{array}{l}8(\mathrm{~N}) \\
1(\mathrm{E})\end{array}$ & $3(\mathrm{~N})$ & $\begin{array}{l}8(N) \\
5(\mathrm{E})\end{array}$ & $4(N)$ & & $\begin{array}{r}13(\mathrm{~N}) \\
1(\mathrm{E})\end{array}$ & 91 \\
\hline Colombia & 6 & $\begin{array}{r}7(\mathrm{~N}) \\
55 \\
\end{array}$ & 55 & $\begin{array}{l}2(\mathrm{~N}) \\
4(\mathrm{E}) \\
\end{array}$ & 18 & $9(\mathrm{~N})$ & 26 & $12(\mathrm{~N})$ & & 56 & 250 \\
\hline Costa Rica & $4(N)$ & $\begin{array}{l}2(\mathrm{~N}) \\
6(\mathrm{E})\end{array}$ & $\begin{array}{l}7(\mathrm{~N})) \\
4(\mathrm{E})\end{array}$ & $\begin{array}{l}2(\mathrm{~N}) \\
1(\mathrm{E})\end{array}$ & $\begin{array}{l}6(\mathrm{~N}) \\
1(\mathrm{E})\end{array}$ & $6(N)$ & $\begin{array}{l}7(N) \\
9(\mathrm{E})\end{array}$ & 3 & $6(N)$ & $4(E)$ & 68 \\
\hline Cuba & & $\begin{array}{l}9(\mathrm{~N}) \\
2(\mathrm{E})\end{array}$ & $\begin{array}{l}1(\mathrm{~N}) \\
1(\mathrm{E})\end{array}$ & & $2(N)$ & & $4(N)$ & $1(N)$ & & $1(E)$ & 21 \\
\hline Dominica & & $2(\mathrm{E})$ & & & $3(\mathrm{E})$ & & 2 & & & $1(E)$ & 8 \\
\hline Ecuador & $\begin{array}{r}4(\mathrm{~N}) \\
12(\mathrm{E})\end{array}$ & $\begin{array}{r}6(\mathrm{~N}) \\
16(\mathrm{E})\end{array}$ & $\begin{array}{r}9(\mathrm{~N}) \\
12(\mathrm{E})\end{array}$ & $\begin{array}{l}1(\mathrm{~N}) \\
1(\mathrm{E})\end{array}$ & $\begin{array}{l}3(\mathrm{~N}) \\
5(\mathrm{E})\end{array}$ & $\begin{array}{l}1(\mathrm{~N}) \\
2(\mathrm{E})\end{array}$ & $\begin{array}{r}5(\mathrm{~N}) \\
14(\mathrm{E})\end{array}$ & & $1(\mathrm{~N})$ & $\begin{array}{l}4(\mathrm{~N}) \\
4(\mathrm{E})\end{array}$ & 100 \\
\hline Granada & & $1(\mathrm{E})$ & & & $6(E)$ & $1(\mathrm{E})$ & $1(\mathrm{E})$ & & & & 9 \\
\hline Guadalupe & & $3(\mathrm{~N})$ & & & $1(\mathrm{~N})$ & $1(\mathrm{E})$ & $1(\mathrm{~N})$ & & & $2(\mathrm{~N})$ & 8 \\
\hline Guatemala & $\begin{array}{r}6(\mathrm{~N}) \\
37(\mathrm{E}) \\
\end{array}$ & $\begin{array}{l}10(\mathrm{~N}) \\
10(\mathrm{E})\end{array}$ & $\begin{array}{l}4(\mathrm{~N}) \\
3(\mathrm{E})\end{array}$ & & 2 & $\begin{array}{l}1(\mathrm{~N}) \\
2(\mathrm{E})\end{array}$ & 38 & $1(\mathrm{E})$ & & 15 & 129 \\
\hline $\begin{array}{l}\text { Guayana } \\
\text { francesa }\end{array}$ & & $4(E)$ & & $1(E)$ & $1(\mathrm{~N})$ & & $2(E)$ & & & $2(\mathrm{~N})$ & 10 \\
\hline Guyana & & $6(E)$ & 2 & $2(E)$ & 1 & & & & & $1(\mathrm{E})$ & 12 \\
\hline Hatí & & $\begin{array}{l}8(N) \\
4(\mathrm{E})\end{array}$ & $2(N)$ & & $\begin{array}{l}1(\mathrm{~N}) \\
2(\mathrm{E})\end{array}$ & 1 & $2(E)$ & & & $2(E)$ & 22 \\
\hline Honduras & $17(\mathrm{E})$ & $\begin{array}{l}1(N) \\
4(E)\end{array}$ & $\begin{array}{l}2(\mathrm{~N}) \\
5(\mathrm{E})\end{array}$ & & $1(\mathrm{E})$ & $\begin{array}{l}1(\mathrm{~N}) \\
3(\mathrm{E})\end{array}$ & $\begin{array}{l}2(\mathrm{~N}) \\
4(\mathrm{E})\end{array}$ & $2(E)$ & & $4(E)$ & 46 \\
\hline $\begin{array}{l}\text { Islas } \\
\text { Cayman }\end{array}$ & & $2(E)$ & $1(\mathrm{E})$ & & $1(\mathrm{E})$ & & & $1(\mathrm{E})$ & & & 5 \\
\hline $\begin{array}{l}\text { Islas } \\
\text { Leeward }\end{array}$ & & 2(E) & $3(E)$ & & $3(E)$ & $1(E)$ & & & & & 9 \\
\hline $\begin{array}{l}\text { Is. Turcos- } \\
\text { Caicos }\end{array}$ & & 1(E) & 1(E) & & & & & & & $1(E)$ & 3 \\
\hline
\end{tabular}


podemos ver las líneas más comunes y conocer la situación de esta especialidad en cada país. Cabe mencionar que este cuadro representa una recopilación preliminar ya que hay mucha literatura en libros, revistas, simposios, tesis o informes que nos remiten a diferentes disciplinas, todas ellas relacionadas con la etnobotánica. Cada país es tratado en forma diferente ya que no hay uniformidad en la revisión de las fuentes. Para México se recopiló una mayor infornıación incluyéndose estadísticas de los congresos mexicanos de botánica, de los simposios dedicados a esta especialidad y de algunas tesis, por lo que se verá desbalanceada la información de México en relación con los otros países.

En los casos de Colombia y Perú se toman datos de simposios y libros sobre este tema. Algo similar se hizo para Ecuador. Los demás países se encuentran en franco proceso de desarrollo de esta disciplina e incluso de varias especia-

Cuadro 1. Continuación.

\begin{tabular}{|c|c|c|c|c|c|c|c|c|c|c|c|}
\hline PAIS & $\begin{array}{l}\text { Arqueo } \\
\text { botánica }\end{array}$ & $\begin{array}{l}\text { Plantas } \\
\text { medi- } \\
\text { cinales }\end{array}$ & $\begin{array}{l}\text { Plantas } \\
\text { comes- } \\
\text { tibles }\end{array}$ & $\begin{array}{l}\text { Estudios } \\
\text { cogno- } \\
\text { sitivos }\end{array}$ & $\begin{array}{l}\text { Aprovecha- } \\
\text { mientos } \\
\text { forestales }\end{array}$ & $\begin{array}{l}\text { Sistemas } \\
\text { agrosil- } \\
\text { vicolas } \\
\text { y huertos }\end{array}$ & $\begin{array}{l}\text { Domesti- } \\
\text { cación y } \\
\text { origen de la } \\
\text { agricultura }\end{array}$ & $\begin{array}{l}\text { Estudios } \\
\text { históricos }\end{array}$ & Mercados & $\begin{array}{l}\text { Gene- } \\
\text { rales }\end{array}$ & Totales \\
\hline $\begin{array}{l}\text { Islas } \\
\text { Virgenes }\end{array}$ & & $3(\mathrm{E})$ & & & & & $1(\mathrm{E})$ & & & $3(E)$ & 7 \\
\hline $\begin{array}{l}\text { Islas } \\
\text { Windard }\end{array}$ & & $2(E)$ & $2(E)$ & & & $1(E)$ & & & & $1(E)$ & 6 \\
\hline Jamaica & & $\begin{array}{l}3(\mathrm{~N}) \\
6(\mathrm{E})\end{array}$ & $\begin{array}{l}1(\mathrm{~N}) \\
3(\mathrm{E})\end{array}$ & $1(\mathrm{E})$ & $\begin{array}{r}2(\mathrm{~N}) \\
12(\mathrm{E})\end{array}$ & & & $3(\mathrm{E})$ & & 2(E) & 33 \\
\hline Martinica & & $1(\mathrm{E})$ & $1(\mathrm{E})$ & & $5(\mathrm{E})$ & $1(\mathrm{E})$ & & 2(E) & & 2(E) & 13 \\
\hline México & $\begin{array}{r}44(\mathrm{~N}) \\
110(\mathrm{E})\end{array}$ & $\begin{array}{r}83(\mathrm{~N}) \\
106(\mathrm{E})\end{array}$ & $\begin{array}{l}70(\mathrm{~N}) \\
49(\mathrm{E})\end{array}$ & $\begin{array}{l}15(\mathrm{~N}) \\
33(\mathrm{E})\end{array}$ & $\begin{array}{l}40(\mathrm{~N}) \\
10(\mathrm{E})\end{array}$ & $\begin{array}{r}100(\mathrm{~N}) \\
49(\mathrm{E})\end{array}$ & $\begin{array}{l}53(\mathrm{~N}) \\
70(\mathrm{E})\end{array}$ & $\begin{array}{l}13(\mathrm{~N}) \\
21(\mathrm{E})\end{array}$ & $\begin{array}{l}\text { 2(N)) } \\
3(\mathrm{E})\end{array}$ & $\begin{array}{l}47(\mathrm{~N}) \\
83(\mathrm{E})\end{array}$ & 1001 \\
\hline Nicaragua & & $\begin{array}{l}1(\mathrm{~N}) \\
5(\mathrm{E})\end{array}$ & $2(E)$ & & $4(E)$ & & $4(E)$ & & & 1(E) & 17 \\
\hline Panamá & $5(\mathrm{E})$ & $\begin{array}{l}2(\mathrm{~N}) \\
7(\mathrm{E})\end{array}$ & $3(E)$ & & $4(E)$ & 2(E) & $7(\mathrm{E})$ & & & $\begin{array}{r}2(\mathrm{~N}) \\
14(\mathrm{E})\end{array}$ & 44 \\
\hline Paraguay & & $\begin{array}{r}12(\mathrm{~N}) \\
3(\mathrm{E})\end{array}$ & $2(\mathrm{~N})$ & $5(\mathrm{~N})$ & $3(E)$ & & $\begin{array}{l}2(\mathrm{~N}) \\
6(\mathrm{E})\end{array}$ & $2(\mathrm{~N})$ & & $\begin{array}{l}4(\mathrm{~N}) \\
5(\mathrm{E})\end{array}$ & 44 \\
\hline Perú & $\begin{array}{l}16(\mathrm{~N}) \\
37(\mathrm{E})\end{array}$ & $\begin{array}{l}14(\mathrm{~N}) \\
45(\mathrm{E})\end{array}$ & $\begin{array}{l}17(\mathrm{~N}) \\
30(\mathrm{E})\end{array}$ & $\begin{array}{r}3(\mathrm{~N}) \\
11(\mathrm{E}) \\
\end{array}$ & $\begin{array}{l}3(\mathrm{~N}) \\
6(\mathrm{E}) \\
\end{array}$ & $\begin{array}{r}6(\mathrm{~N}) \\
15(\mathrm{E}) \\
\end{array}$ & $\begin{array}{l}36(\mathrm{~N}) \\
27(\mathrm{E})\end{array}$ & $\begin{array}{r}10(\mathrm{~N}) \\
6(\mathrm{E}) \\
\end{array}$ & & $\begin{array}{l}13(\mathrm{~N}) \\
18(\mathrm{E})\end{array}$ & 313 \\
\hline Puerto Rico & & $\begin{array}{l}4(\mathrm{~N}) \\
9(\mathrm{E})\end{array}$ & $\begin{array}{l}3(\mathrm{~N}) \\
7(\mathrm{E})\end{array}$ & & $2(E)$ & & & $1(E)$ & & $5(\mathrm{E})$ & 31 \\
\hline $\begin{array}{l}\text { República } \\
\text { Dominicana }\end{array}$ & & $\begin{array}{l}1(\mathrm{~N}) \\
5(\mathrm{E})\end{array}$ & $1(\mathrm{E})$ & & $1(E)$ & & 2(E) & $1(\mathrm{E})$ & & $\begin{array}{l}2(\mathrm{~N}) \\
1(\mathrm{E})\end{array}$ & 14 \\
\hline El Salvador & $6(E)$ & $\begin{array}{l}1(\mathrm{~N}) \\
3(\mathrm{E})\end{array}$ & $1(\mathrm{E})$ & & $1(\mathrm{E})$ & & $3(E)$ & & & $\begin{array}{l}1(\mathrm{~N}) \\
4(\mathrm{E})\end{array}$ & 20 \\
\hline Surinam & & $\begin{array}{l}2(\mathrm{~N}) \\
6(\mathrm{E})\end{array}$ & $\begin{array}{l}1(\mathrm{~N}) \\
4(\mathrm{E})\end{array}$ & $2(E)$ & $2(E)$ & & $1(E)$ & $1(\mathrm{E})$ & & & 19 \\
\hline $\begin{array}{l}\text { Trinidad } \\
\text { Tobago }\end{array}$ & & $\begin{array}{l}1(\mathrm{~N}) \\
6(\mathrm{E})\end{array}$ & 2(E) & & $\begin{array}{l}1(\mathrm{~N}) \\
7(\mathrm{E})\end{array}$ & & $\begin{array}{l}2(\mathrm{~N}) \\
8(\mathrm{E})\end{array}$ & & & 2(E) & 29 \\
\hline Uruguay & & & & $1(\mathrm{~N})$ & & & $2(\mathrm{~N})$ & & & $1(\mathrm{~N})$ & 4 \\
\hline Venezuela & $\begin{array}{l}1(\mathrm{~N}) \\
4(\mathrm{E})\end{array}$ & $\begin{array}{r}3(\mathrm{~N}) \\
13(\mathrm{E})\end{array}$ & $\begin{array}{l}4(\mathrm{~N}) \\
8(\mathrm{E})\end{array}$ & $2(E)$ & $\begin{array}{l}3(\mathrm{~N}) \\
1(\mathrm{E})\end{array}$ & & $\begin{array}{l}4(\mathrm{~N}) \\
4(\mathrm{E})\end{array}$ & $3(\mathrm{~N})$ & & $\begin{array}{r}3(\mathrm{~N}) \\
10(\mathrm{E})\end{array}$ & 63 \\
\hline TOTALES & 361 & 591 & 392 & 100 & 197 & 220 & 439 & 91 & 7 & 370 & 2768 \\
\hline
\end{tabular}

(N) Investigador Nacional

(E) Investigador Extranjero
América del Sur

Centroamérica

Caribe

México 
lidades de la botánica. También hay países en los que la botánica está muy desarrollada, pero con poco impulso o interés en la etnobotánica; tales son los casos de Chile, Costa Rica, Argentina y Venezuela.

Las revistas donde se encuentran la mayoría de estudios etnobotánicos son, en orden de prioridad: Economic Botany, Journal of Ethnopharmacology, Journal of Ethnobiology, Advances in Economic Botany; éstas son las fuentes primarias de los datos presentados en el Cuadro 1.

Revistas botánicas o de agronomía donde aparecen ocasionalmente estudios etnobotánicos son: Bulletin of the Torrey Botanical Club, Botanical Museum Leaflets of Harvard, Annals of Missouri Botanical Garden, Memories of New York Botanical Garden, American Journal of Botany, Canadian Journal of Botany, Tropical Agriculture, New Crops, World Crops y Euphytica. Revistas como Ethnology, Current Anthropology, Annual Reviews of Ecology, Botany y Anthropology, American Anthropologist, Ethnohistory, Hispanic-American Historical Review, American Antiquity, Archaeology y Journal of Agricultural History, son fuentes obligadas en el rastreo bibliográfico de estudios etnobotánicos. Finalmente, hay diversos libros, folletos, memorias, congresos, tesis e informes de investigación en los que también hay bastante información sobre el tema.

\section{SITUACIÓN ACTUAL DE LA ETNOBOTÁNICA MEXICANA}

Empezaremos por indicar que en México se llevan a cabo diferentes investigaciones, siendo los estudios sobre plantas medicinales los más abundantes, desde los muy descriptivos y que se limitan a listados florísticos hasta los de tipo comparativo (Aguilar et al., 1994a, b;. Aguilar et al., 1994c; Argueta, 1994; Argueta y Zolla, 1994; Mellado, 1994;Zolla, 1994).

Aguilar et al. (1994b) hacen un estudio comparativo entre etnias y usos de las mismas especies o diferentes taxa, siguiendo dicha comparación bajo condiciones ecosistémicas similares y niveles culturales más o menos semejantes. Bye analiza la información etnobotánica con relación a la composición fitoquímica de una misma especie colectada en diferentes sitios.

Siguen los trabajos sobre intentos de domesticación y cultivos de plantas medicinales, así como su distribución geográfica (Estrada, 1994). Los estudios sobre flórulas medicinales hechos con diversos médicos indígenas pertenecientes a varias etnias se deben a Aguilar et al. (1994c). Investigaciones exhaustivas sobre bibliografía y trabajo de campo combinados dan por resultado la flora médica, la elaboración de un atlas de plantas medicinales para México por Argueta (1994) y un diccionario de medicina tradicional y herbolaria por Zolla (1994); Mellado (1994) hace el estudio sobre medicina tradicional de los pueblos indígenas de México. Estas son en mi opinión algunas investigaciones notables en esta línea de investigación etnobotánica.

Otra línea de investigación muy popular es la del estudio de las plantas comestibles. En ella también se busca desde la descripción de los usos comestibles o un listado de plantas, hasta el conocimiento de la composición bromatológica de los alimentos vegetales y su incorporación a la dieta cotidiana de las comunidades estudiadas. Existen tanto estudios teóricos como aplicados y a nivel comunitario o comparativo entre regiones (Ysunza etal. (en prensa), Villalobos (1994), Caballero (1994). El último autor hace incluso uno de los primeros y más completos trabajos cuantitativos para la etnobotánica nacional.

Los trabajos sobre domesticación, cultivos y recursos fitogenéticos de recursos mexicanos son muy completos desde el punto de vista teórico y metodológico, y en varios de ellos se hace un análisis histórico muy amplio, así como de su conservación y/o su manejo. Destacan los siguientes trabajos: para agaves o recursos fitogenéticos de la milpa, (Colunga y May-Pat, 1992,1993); en palma de coco (Zizumbo, 1993a y b); palma sabal (Caballero, 1994); maíz (Sánchez y Goodman 1992 ); en frijol (Basurto et al., en prensa), en guaje (Casas, 1992); en pitaya (Pimienta y Nobel, 1994, Castillo, 1991). Esta área de la etnobotánica va en continuo aumento y con gran diversidad de enfoques, y se cuenta también con buenos estudios de naturaleza cuantitativa y de alta calidad académica.

Con relación a problemas de domesticación y taxonomía o ecología hay un estudio integral entre estas disciplinas por Bye et al. (1993), Rico-Gray et al. (1990) y Bellón (1991, 1994). Otro proyecto que se inicia en 1995 y será muy novedoso al integrar diversas especialidades de la agronomía y la botánica, es el de conservación in situ de cultivos mesoamericanos: maíz, frijol, chile y calabaza. Dentro de este tipo de estudios la colaboración se da incluso a nivel internacional, en virtud de que existe apoyo por parte de fundaciones y organismos internacionales, lo cual le da otra dimensión a la botánica mexicana (Bye et al., en proceso).

Las investigaciones en etnobotánica histórica no se desarrollan todavía, hay varios historiadores y algunos botánicos ocupados en estudiar usos pasados de las plantas y cultivos marginados, pero los avances son pocos. Como ejemplo de estos estudios están: Martínez et al. (1992); Debouck (1992); Cuevas (1992); Lira y Montes (1992); Ortega, et al. (1993).

Otra línea que se desarrolla con vigor dentro de la etnobotánica nacional es la de estudios sobre percepción de la naturaleza y sistemas de clasificación popular o folk. Por lo general se trata de estudios a los que el botánico aporta poco, pues más bien son trabajos de antropólogos o lingüistas con ayuda de botánicos (Breedlove y Laughlin, 1993; Caballero, 1994). Casas et al. (1994) investigan problemas etnobotánicos de conservación de recursos vegetales en la Mixteca Guerrerense.

Este es el panorama de los principales proyectos, tesis, artículos o libros que muestra el estado actual de la etnobotánica mexicana.

Ahora que se comenta brevemente hacia donde se proyecta la etnobotánica del país, tal vez no se piense que esta especialidad de la botánica va ganando espacios de 
docencia e investigación; hay capacitación a muy bajo nivel, y los pocos especialistas que se están formando no tienen bases muy sólidas. Además, aunque hay estudiantes y tesistas, pocos continúan en el campo y cambian de actividad. Por ello es de mucha ayuda el interés del investigador por formar más y mejores recursos humanos.

Con relación a los estudios en esta materia, hay mucha gente trabajando con entusiasmo, pero tieñn pucos recursos para lograr avances notables y se fomenta el desarrollo de una etnobotánica descriptiva, anecdótica y sin capacidad de cambiar el rumbo. En las pocas instituciones educativas que tienen cursos e investigaciones etnobotánicas, por lo general no hay infraestructura para avanzar en la calidad de la enseñanza o investigación. El carácter multidisciplinario de la etnobotánica provoca de manera natural la selección y tarde o temprano muchos grupos quedarían al margen del desarrollo científico contemporáneo. La UNAM, la UACH y el Colegio de Postgraduados de Montecillos, Edo. de México, son las instituciones con posibilidades de lograr el impulso futuro de la disciplina; en ellas se tienen mayores posibilidades de renovar y dirigir hacia nuevos enfoques el trabajo etnobotánico. Las demás instituciones, con los escasos apoyos que tuvieron en cierto momento, no avanzan. Solamente la animación de los especialistas de esas instituciones mantiene vivo el interés en este campo. Otras pueden o podrían ofrecer un buen espacio, pero esto depende mucho de la política gubernamental y del momento por el que pasa la institución. Además no han logrado obtener apoyos extraordinarios, los cuales sí tienen las tres instituciones antes citadas.

Como se demandan enfoques multidisciplinarios en el estudio, manejo, conservación y educación sobre recursos vegetales, la etnobotánica facilita mucho tal enfoque globalizador. Por otro lado, hay más apoyos de todo tipo para el trabajo multidisciplinario, los cuales son parte de los paradigmas biológicos actuales; de ahí la popularidad y expansión de nuestra especialidad. Los gobiernos, organizaciones civiles, las sociedades de profesionistas, están alertas y animan tales campos de estudio. Citaré ejemplos de lo que puede hacerse en el futuro: Las investigaciones etnoecológicas se verían beneficiadas con el aporte serio y continuo de la etnobotánica, pudiendo delimitar mejor los ambientes ecológicos y sociales para así planificar ecológicamente una zona. El vínculo con economistas especializados en economía de recursos naturales puede ayudar a resolver la división entre economía y botánica, optando por aquellos proyectos de desarrollo socioeconómico en que se integren bien las teorías y métodos de economistas y botánicos.

La planificación para un buen manejo de los ecosistemas, la delimitación de ecosistemas para manejo, la conservación y los programas de educación ambiental serían más exitosos si se unen los esfuerzos de ecólogos vegetales y etnobotánicos. Si deseamos que avancen las ciencias botánicas y tengan cada vez más injerencia en la toma de decisiones, se deberán promover trabajos de equipo en los que el propio botánico apoya a otros, y en los que no se descalifiquen ciertas áreas tan rápido y a la ligera. Espero que la etnobotánica pueda contribuir en este aspecto tan común en nuestros círculos académicos.

Corresponde a cada investigador especializado en un campo de la botánica el futuro de su especialidad, agregar discursos no sirve de nada. Ahora está clara la necesidad de mejorar en todos los aspectos que comprende una investigación o la planeación de un curso que se desea impartir y que plantea búsquedas al interior de nuestra especialidad botánica. Y es clara la necesidad de mejorar los temas, la bibliografía y las prácticas de los cursos de etnobotánica. A nivel personal confío y espero que avancemos hacia trabajos de equipo y a la promoción de discusiones, seminarios y el uso de la tribuna de nuestra Sociedad Botánica para discutir y planear acciones en las que la botánica sea la punta de lanza de las decisiones. Espero que no seamos más espectadores, sino los actores centrales en las políticas sobre el manejo de los recursos vegetales. Así podremos ver cuáles proyectos son prioritarios en el desarrollo futuro y actual de la botánica nacional.

Finalizo con la idea de que únicamente los proyectos viables, es decir los que reciben apoyo de cualquier tipo, son los que indican la importancia y utilidad de nuestro campo. Además las publicaciones y los materiales de difusión son la mejor prueba de que algo es necesario y digno de apoyo.

El comentario del Dr. Lot en el último congreso de Botánica no debe ser olvidado. Es en la etnobotánica en donde se demanda un mayor y rápido esfuerzo por hacer públicos tantos estudios ya concluidos o con buenos avances. Sólo así tendremos oportunidad de ser confrontados y de que se conozca nuestro potencial para el desarrollo de una ciencia de calidad.

\section{LA CUANTIFICACIÓN EN LOS ESTUDIOS ETNOBOTÁNICOS}

Antes de presentar una idea general de las prácticas estadisticas más utilizadas en la etnobotánica, cabe comentar que en algunos de los trabajos revisados que utilizan tales técnicas, los investigadores no contaban con un problema bien planteado, lo cual es forzoso en el análisis cuantitativo de cualquier investigación. La estadística tiene sentido y utilidad cuando el etnobotánico tiene un trabajo bien estructurado en sus objetivos, temas a investigar, resultados que espera alcanzar y predicciones claras acerca de dónde incidirá su trabajo.

Hay una relación directa entre la realidad estudiada, el diseño y la estructura de la investigación y la valoración en el análisis de los datos, lo que se observa en los trabajos que a continuación se comentarán. Es importante la elección de las variables y la forma de análisis de los resultados. Entre las variables que han sido estudiadas por los etnobotánicos están: los datos de las plantas en su biología, ecología o genética; composición química; usos; plantas utilizadas para atacar diferentes tipos de enfermedades o la misma enferme- 
dad; tipos de alimentos; conservación y/o manejo de una especie.

Durante los últimos 10 años se han desarrollado estudios con más orientación ecológica que buscan la cuantificación de los resultados con los siguientes enfoques:

1) Se muestrea una parcela, cuadrante o transecto, y se cuentan las veces que una persona explota un individuo o una especie. Se observa si es utilizada para el mismo uso, uso diferente o si deja de utilizarse, se levanta la información etnobotánica y se corroboran los datos levantados con un segundo muestreo. La mayoría de este tipo de estudios se han llevado a cabo en selvas tropicales húmedas. Hasta ahora en muchos estudios con este enfoque se desdeñan arbustos y hierbas, por lo tanto nos dan un cuadro muy desviado de lo es el uso de las plantas en este tipo de ecosistemas (Flores, 1983, 1988; Balée, 1986,1987; Unruh, 1986; Boom, 1987; Denevan y Treacy, 1988; Unruh y Flores, 1988; Milliken et al.,1992).

2) Se utilizan hipótesis estadísticas, siendo loable el intento de cuantificar aspectos que a veces son difíciles de analizar con técnicas estadísticas. Dentro de este enfoque se encuentran trabajos sobre una especie en particular, floras útiles y cambios en los usos de plantas debido a los cambios culturales.

Cada vez que se entrevista a una persona y/o cada uso que informa el entrevistado se denomina eventos. A partir de los eventos se conoce el valor de uso por especie, graficando el número de usos y el número de especies utilizadas. De aquí se obtienen distribuciones logarítmicas normales, con una línea tenue o velada: Se entiende que a mayor número de especies utilizadas habrá menor número de usos por especie y viceversa. La primera situación es común en regiones con gran diversidad florística y un gran conocimiento de las plantas por los grupos humanos que ahí habitan. Es la situación en etnias de las selvas tropicales húmedas o secas.

Recientemente, en un estudio de este tipo hecho en el Amazoǹas Peruano por Phillips y Gentry (1993), se sugieren fórmulas específicas para medir el valor de uso por especie. Con la propuesta de estos autores se pueden probar diversas hipótesis nulas, las cuales permiten afinar los conocimientos de cada informante principalmente en correlaciones entre densidad/frecuencia, tamaño y utilidad, las frecuencias de uso por tipo de vida, familias botánicas, edad, sexo e intereses personales en lo concerniente al conocimiento por niveles de discriminación que tenga el informante.

Otros estudios han utilizado pruebas estadísticas ya conocidas, (paramétricas y no-paramétricas) para conocer por ejemplo, los efectos de interacción cuantitativa y predecir la eficacia de una planta medicinal (Johns, Kokwaro y Kimanani, 1990).
3) Algunos estudios han utilizado técnicas de análisis multivariado como análisis de conglomerados, análisis de componentes principales, análisis de coordenadas principales o análisis discriminante. Los análisis multivariados tienen la gran ventaja de poder analizar el comportamiento de más de una variable a la vez. Estos análisis estadísticos se han utilizado, por ejemplo, en el estudio de la estructura de huertos familiares (Caballero, 1994), en el consumo de plantas alimenticias (Ríos, 1994), en el análisis de tres poblaciones de Leucaena esculenta (Casas, 1992)

Con base en los ejemplos citados se puede resumir que cada vez es mayor la necesidad de utilizar técnicas que cuantifiquen los datos etnobotánicos porque ofrecen las siguientes ventajas: a) Se controla mejor la información y se pueden agrupar los datos con claridad para diversos propósitos de investigación o aplicación de los resultados. b) Se agilizan los estudios y se tiene mayor objetividad dentro de los estudios etnobotánicos modernos. c) Se perciben y aclaran mejor los elementos subjetivos que tan fuertemente inciden en el trabajo etnobotánico, los cuales pueden provocar distorsiones por parte del informante o del investigador. d) El método estadístico y sus técnicas permiten mejorar la teoría y práctica del botánico y por tanto llegar a niveles de investigación más finos. También permiten convertir a la etnobotánica en un campo de la ciencia moderna con la intención de servir a un número mayor de investigadores y a la gente con quien trabaja el etnobotánico.

De acuerdo al análisis que hace de la estadística y la filosofía de la ciencia el Dr. Méndez, destacado investigador mexicano en estadística (Méndez, 1993) existen las siguientes limitantes en la aplicación de la estadística. En primer lugar, dentro de la corriente del pensamiento científico positivista, el problema a investigar sólo tiene valor o utilidad si se cuantifica. Esto es más común en las ciencias naturales. En segundo lugar, en la investigación etnobotánica moderna hay gran variabilidad o variación, como ocurre con otras ciencias naturales y sociales. Por medio de sus modelos, la estadística nos ayuda a valorar y analizar la información, pero en campos donde hay tanta inferencia, ésta es central para poder aplicar correctamente la estadística. Me pregunto qué tanta formación filosófica tienen quienes cuantifican, pues para asegurar que su propuesta es la mejor deben mostrar diseños y estructuras sólidos de sus investigaciones. Cuando menos no veo eso en científicos norteamericanos y europeos cuya única preocupación es tener el consenso social de sus colegas y de los tomadores de decisiones en políticas científicas. No es aquí el lugar adecuado para discutirlo, pero hay una fuerte crítica a esta postura en la ciencia moderna.

En tercer lugar, la era científica de la cual nos sentimos orgullosos está en crisis, y filósofos-científicos tan preclaros como B. Russell indican que así como la era teológica se derrumbó, la ciencia tiene el riesgo de tomar el mismo cauce cuando se le constriñe a ciertas posturas 
filosóficas que van de acuerdo con el modelo y política dominante, cayendo en dogmas al igual que la religión. En cuarto lugar, hay ciertos hechos que no pueden ser verificables, y ejemplos de ellos los tenemos en abundancia en la biología. La corriente positivista le da mucha importancia a la verificación, objetividad, racionalidad y empiria. Estas cualidades permiten que surjan métodos científicos, y como muchos incluyen dentro de los métodos a la estadística, se va produciendo una excesiva confianza en la ciencia, particularmente en las matemáticas y, dentro de ellas, en la estadística. «El hecho de que una hipótesis produzca una predicción que es verificada en la práctica no establece la validez de la hipótesis, a esto Popper le llama FALSACIONISMO, no podemos mostrar que una hipótesis es cierta, pero si podemos rechazarla o demostrar que es falsa; de aquí la posición no importa cómo se inventan las hipótesis y someten a pruebas rigurosas, las que subsistan más son las adecuadas; así avanza la ciencia» (Méndez, 1993).

Finalmente. hasta el momento una gran cantidad de estudios cuantificados no dan solución a las demandas sociales de la gente investigada, se predice bien y se resuelve mal. Hay estudiosos de la ciencia moderna que le dan muchas vueltas a esta parte de la etnobotánica. Critican mucho a este tipo de ciencia y cuando recurren a las cifras frías ven que la razón científica occidental no requiere involucrarse más en lo social ya que esto es subjetivo. Esta es la filosofía del investigador y no cae en lo científico.

Ante esta situación, como indica el autor citado, se debe ser precavido y autoanalítico sobre nuestro papel social y científico, los científicos comparten ambas categorías y hay buena voluntad e interés por atenderlas. Es necesario dar el mismo valor a las diferentes posturas en la ciencia, evitar los prejuicios y aceptar las mejores vías o propuestas que nos ofrecen nuestras investigaciones. Entre ellas está en un lugar privilegiado la estadística, usémosla para ser mejores investigadores, pero no para calificar sí es o no la mejor opción cuando no la sigan algunas personas.

\section{AGRADECIMIENTOS}

Las sugerencias y criticas al presente trabajo fueron hechas en el Jardín Botánico del Instituto de Biología por mis colegas etnobotánicos Dr. Javier Caballero, del Laboratorio de Etnobotánica, Dr. Robert Bye, Director del Jardín Botánico. A la Biól. Virginia Evangelista O., por sus comentarios y observaciones al texto y al M. en C. Alejandro Casas, becario del Instituto de Biología mis más reconocidos agradecimientos por su amable revisión al presente trabajo. Los errores y prejuicios o valoraciones son responsabilidad total del autor.

\section{LITERATURA CITADA}

Aguilar A, Camacho JR, Chino S, Jácquez P, Lopez ME. 1994a. Herbario medicinal del Instituto Mexicano del Seguro Social. Información etnobotánica. IMSS, México, D. F.
Aguilar A, Camacho JR, Chino S, Jácquez P, Lopez ME. 1994b. Plantas medicinales del Herbario IMSS: Cuadros básicos por aparatos y sistemas del cuerpo humano. IMSS, México, D. F.

Aguilar A, Argueta A, Cano L (Coords.). 1994c. Flora medicinal indígena de México. INI, México D. F. 3 vols.

Argueta A. 1994. Atlas de las plantas de la medicina tradicional mexicana. INI, México, D.F. 3 vols.

Argueta A, Zolla C (Coords.) 1994. Nueva bibliografía de la medicina tradicional mexicana. INI, México, D. F.

Baleé W. 1986. Informe preliminar sobre inventario forestal e a etnobotanica Kaapor(MA). Bol. Mus. Paraense Emílio Goeldi, Ser. Bot. 2: 141-167.

Baleé W. 1987. A etnobotánica quantitativa dos Indios Tembé (Río Gurupi, Pará). Bol. Mus. Paraense Emílio Goeldi, Ser. Bot. 3: 29-50.

Basurto P FA. 1982. Huertos familiares en dos comunidades nahuas de las Sierra Norte de Puebla: Yancuictlalpan y Cuauhtapanaloyan. Tesis de Licenciatura, Facultad de Ciencias, UNAM. México, D. F.

Basurto P FA, Martínez D, Castellanos A, Martínez MA. Ciclo agrícola y fenología del Phaseolus coccineus L., en sistemas de agricultura tradicional de la Sierra Norte de Puebla. Etnoecológica 4: (en prensa).

Bellón MR.1991. The Ethnoecology of maize variety management: A case study from Mexico. Human Ecol. 19: 389-418.

Bellón MR, Brush SB. 1994. Keepers of maize in Chiapas, Mexico. Econ. Bot. 48: 196-209.

Boom BM. 1987. Ethnobotany of the Chácobo Indians, Bení, Bolivia. Advances in Economic Botany, Vol.4. The New York Botanical Garden, Bronx, NY.

Breedlove DE, Laughlin RM. 1993. The flowering of Man. A tzotzil botany of Zinacantan. Smithsonian Contributions to Anthropology. No. 35. Smithsonian Institution Press, Washington D. C. 2 vols.

Bye R, Delgado A, Piñero D, Castellanos A, Palomino G, Gepts P, Martínez MA, González de León D. 1993. Molecular, cultural and ecological markers of genetic diversity in Mexican species of beans (Phaseolus). Reportes técnicos I-III. AID, Washington, D.C.

Bye R, Qualset C, Delgado A, Acosta J, Gepts P, Castillo F, Ortega PR, Goodman M, Martínez MA. Proyecto sobre conservación in situ de cultivos mesoamericanos. Fundación McKnight, Wisconsin (en proceso).

Caballero J. 1994. Use and management of sabal palms among the Maya of Yucatan. Ph. D. Thesis. Department of Anthropology, University of California, Berkeley.

Casas F A.1992. Etnobotánica y procesos de domesticación en Leucaena esculenta (Moc. et Sessé ex A. DC.) Benth.Tesis de Maestría, Facultad de Ciencias, UNAM. México, D. F.

Casas F A, Viveros JL, Caballero J. 1994. Etnobotánica Mixteca: Sociedad, cultura y recursos naturales en la Montaña de Guerrero. CONACULTA-INI. México, D. F.

Colunga GM P, May-Pat F. 1992. El sistema milpero y sus recursos fitogenéticos. En: Zizumbo D, Rasmussen CR, Arias LM, Terán S, edrs. La modernización de la milpa en Yucatán: utopía o realidad. CICY y DANIDA, Mérida, Yucatán, 97134.

Colunga GM P, May-Pat F. 1993. Agave studies in Yucatan, Mexico. I. Past and present germplasm diversity and uses. Econ. Bot. 47: 312-327.

Colunga-GM P, Coello-C J, Espejo-P L, Fuente-M L. 1993. Agave studies in Yucatan, Mexico. II. Nutritional value of the inflorescence peduncle and incipient domestication. Econ. Bot. 47: 328-334. 
Cuevas-J A. 1992. Jocote, ciruelo (Spondias purpurea). En: Cultivos marginados, otra perspectiva de 1492. FAO-NU, Roma, 109113.

Debouck DG. 1992. Frijoles (Phaseolus spp.). En: Cultivos marginados, otra perspectiva de 1992. FAO-NU, Roma, 45-60.

Del Castillo-R F, Trujillo S. 1991. Ethnobotany of Ferocactus histrix and Echinocereus platyacanthus (Cactaceae) in the semiarid Central Mexico: Past, present and future. Econ. Bot. 45: 495502.

Denevan WM, Treacy JM. 1988. Young managed fallows at Brillo Nuevo. En: Denevan WM, Padoch C, edrs. Swidden-fallow agroforestry in the Peruvian Amazon. Adv. Econ. Bot. 5: 8-46.

Estrada E. 1994. Plantas medicinales de México. UACH, Chapingo, Edo. de México.

Flores-P S. 1983. Agroforestería en la Amazonía Peruana: Investigaciones en marcha de la Universidad Nacional de la Amazonía Peruana. Centro Agronómico Tropical de Investigación y Enseñanza (CATIE), Departamento de Recursos Naturales Renovables, Turrialba.

Flores-P S. 1988. Old managed fallows at Brillo Nuevo. En: Denevan WM, Padoch C, edrs.. Swidden-fallow agroforestry in the Peruvian Amazon. Adv. Econ. Bot. 5: 53-66.

Harré R. 1970. The principles of scientific thinking. University of Chicago Press, Chicago.

Holt JE, Schoorl D. 1993. Redefining institutional roles in agricultural reseach organizations direction for cultural change. Agr. Syst. 41: 77-92.

Johns T, Kokwaro JO, Kimani EB. 1990. Herbal remedies of the Luo of Siaya District, Kenya: Establishing quantitative criteria for consensus. Econ. Bot. 44: 369-381.

Koyré A. 1983. Estudios galileanos y otros ensayos en filosofía de las ciencias. Guadarrama, Madrid.

Kuhn TS. 1979. The structure of scientific revolutions. International Encyclopedia of Unified Science, 2nd. ed. University of Chicago Press, Chicago.

Lira-S R, Montes S. 1992. Cucúrbitas (Cucurbita spp.). En: Cultivos marginados, otra perspectiva de 1492. FAO-NU, Roma, 61-75.

Martínez-A MA. 1991. La etnobotánica en Latinoamérica. Memorias del 3er Simposio Colombiano de Etnobotánica, INCIVA, Cali, 1-14.

Martínez-A MA, Ortega-P R, Cruz-L A. 1992. Repercusiones de la introducción de la flora del Viejo Mundo a América, y causas de la marginación de los cultivos. En: Cultivos marginados, otra perspectiva de 1492. FAO-NU. Roma, 23-33.

Martínez-A MA, Evangelista V, Mendoza M, Morales G, Toledo G, Wong A. 1995. Catálogo de plantas útiles de la Sierra Norte de Puebla. Cuadernos del Instituto de Biología. IB, UNAM, México, D.F.

Mellado CV, coord. 1994. La Medicina tradicional de los pueblos indígenas de México. INI, México, D. F. 3 vols.
Méndez ZI. 1993. Filosofía de la ciencia y estadística. En: De la Fuente J., Ortega R, Sámano M, coords. Agricultura y Agronomía en México, 500 años. Difusión Cultural de la UACH, Chapingo, Edo. de México, 13-32.

Ortega PR, Cruz A, Martinez MA. 1993. Procesos y causas de la marginación de cultivos introducidos. En: De la Fuente J., Ortega R, Sámano M, coords. Agricultura y Agronomía en México, 500 años. Difusión Cultural de la UACH, Chapingo, Edo. de México, 141-154.

Milliken W, Miller RP, Pollard SR, Wandelli EV. 1992. Ethnobotany of the Waimiri-Atroari Indians. Royal Botanic Gardens, Kew.

Phillips O, Gentry AH. 1993a. The useful plants of Tambopata, Peru: I. Statistical hypothesis test with a new quantitative technique. Econ. Bot. 47: 15-32.

Phillips O, Gentry AH. 1993b. The useful plants of Tambopata, Peru: II. Additional hypothesis testing in quantitative ethnobotany. Econ. Bot. 47: 33-43.

Pimienta E, Nobel PS. 1994. Pitaya (Stenocereus spp. Cactaceae): an ancient and modern fruit crop of Mexico. Econ. Bot. 48: 76-83.

Rico-Gray V, García F JG, Chemac A, Puch A, Sima P. 1990. Species composition, similarity and structure of Mayan homegardens in in Texpenal and Tixcacaltuyub, Yucatan, Mexico. Econ. Bot. 44: 470-487.

Ríos M. 1994. Tendencias de cambio en el aprovechamiento de las plantas comestibles no cultivadas en la Amazonía Ecuatoriana. Tesis de Maestría, Facultad de Ciencias, UNAM. México, D.F.

Sánchez JJ, Goodman MM. 1992. Relations among the Mexican Races of Maize. Econ. Bot. 46: 72-85.

Unruh J. 1986. Ecological aspects of swidden-fallow management in the Peruvian Amazon. Ms. Thesis. University of Wisconsin, Madison.

Unruh J, Flores PS. 1988. Relative abundance of the useful component in old managed fallows at Brillo Nuevo. En: Denevan WM, Padoch C, edrs. Swidden-fallow agroforestry in the Peruvian Amazon. Adv. Econ. Bot. 5: 67-73

Villalobos G. 1994. Plantas comestibles en dos comunidades de la Sierra Norte de Puebla: Xochitlán de Vicente Suárez y Zapotitlán de Méndez. Tesis de Licenciatura, FES-Zaragoza,UNAM. México, D. F.

Ysunza OA, Diez US, Martínez-A MA, Martínez ME, López NL, Contreras A, Salas V Monserrat. El CECIPROC y el desarrollo nutricional en Oaxaca (en prensa)

Zizumbo D, Hernández F, Harries HC. 1993a. Coconut varieties in Mexico. Econ. Bot. 47: 65-78.

Zizumbo D, Arellano J, Lira J, Xix G, Pech O. 1993b. Establecimiento de una colección de germoplasma de Cocos nucifera L. en la costa norte de Yucatán. En: Libro de resúmenes del XII Congreso Mexicano de Botánica, SMB y UAY. Mérida, Yuc. p. 278.

Zolla C. 1994. Diccionario enciclopédico de la medicina tradicional mexicana. INI, México, D. F. 2 vols. 